\title{
DETRÁS DEL TEXTO: NOTAS SOBRE EL FONDO FILOSÓFICO DE LA SEMIÓTICA DE TARTU (ARTÍCULO PRIMERO)
}

\author{
Mijaíl I. Lotman \\ Universidad de Tartu, Estonia \\ (Traducción del ruso de Rafael Guzmán)
}

0.0. En el presente trabajo quiero detenerme en algunos aspectos fundamentales del enfoque hacia el texto que fue formulado en los trabajos de mi padre, el profesor Iuri Mijailovich Lotman, y que estuvo en la base de la así llamada escuela semiótica de Tartu-Moscú.

Una particularidad de la escuela estructural-semiótica de Tartu es su textocentrismo: no es la lengua, ni el signo, ni la estructura, ni las oposiciones binarias, ni las reglas gramaticales, sino el texto el que es el centro de su sistema conceptual. A la escuela de Tartu se le ha dedicado un número significativo de publicaciones, que analizan desde diferentes puntos de vista su proceso de formación y su historia, sus postulados teóricos fundamentales ${ }^{1}$. La particularidad de mi trabajo consis-

1 Mencionemos solamente dos monografías: A. Shukman. Literature and Semiotics. 
te en que voy a hablar no de los postulados que fueron manifestados en diferentes publicaciones sobre el texto, sino sobre los que quedaron sin enunciar o insuficientemente articulados. En otras palabras, me interesan no tanto los textos de Tartu sobre el texto, cuanto lo que ha quedado fuera de estos textos, detrás de ellos.

0.1. Otra observación previa más. La metodología moderna de la ciencia exige fijar la posición del investigador en relación con el objeto investigado por él. En este sentido, mi posición plantea no sólo una serie de incomodidades (en primer lugar, de carácter psicológico), sino también, al parecer, una considerable ventaja. Por un lado, tuve la suerte de ser testigo del nacimiento, del proceso de formación y desarrollo de las normas teóricas y metodológicas de la escuela semiótica de Tartu. Mi propio proceso de formación como especialista está indisolublemente relacionado con este contexto: no tuve que aprender la lengua de la Escuela de Tartu; es, por decirlo así, mi lengua científica «natal». Por otro lado, procuré distanciarme, especialmente al principio y en la medida de lo posible, del carisma de mis padres y buscar mis propios caminos (me esforcé por irme del ambiente acentuadamente humanístico de los estudios de Tartu a zonas más «precisas» de la métrica, de la lingüística, de la cibernética); incluso geográficamente intenté separarme, trasladándome de Tartu a Tallinn. Y aunque más tarde aparecieron también algunas publicaciones, firmadas por mi padre y por mí, en realidad no fueron resultado de esfuerzos conjuntos: cada una de ellas se dividen con bastante precisión en partes, escritas por cada uno de los autores. Todo esto crea las premisas para la posibilidad de un efecto «estereoscópico»: voy a intentar analizar el problema desde el punto de vista interno y externo de manera simultánea.

1.0. I. M. Lotman no sólo no manifestó ningún interés por la argumentación filosófica y metodológica de sus concepciones, sino que incluso procuró ocultarlas. Por supuesto, esto estaba relacionado, en cierta medida, con la prensa ideológica: el estructuralismo de la escuela de Tartu encajaba mal en el dogma marxista (Compárese la Introducción a las Lecciones de poética estructural ${ }^{2}$ ). Pero no se trata sólo

A study of the writings of Yu. M. Lotman. Amsterdam, U. A. 1977 y P. Grzybek. Studien zum Zeichenbegriff der sowjetischen Semiotik (Moskauer und Tartuer Schule). Bochum, 1989.

${ }_{2}$ Yu. M. Lotman. Lektsii po strukturalnoj poetike. Vyp. 1: (Vvedenie. Teoria stija). Trudy po znakovym sistemam I (TRÜ toim., \#160). Tartu, 1964. 
de esto, sino de la actitud de I. M. Lotman hacia la propia filosofía. Considera imprescindible advertirlo, en su obra seguramente más filosófica, con las siguientes palabras:

Esta breve exposición de algunos principios investigadores que se presenta al lector no pretende tener un significado filosófico. El autor está muy lejos de pretensiones de este tipo ${ }^{3}$.

Conviene subrayar que el distanciamiento de I. M. Lotman de la filosofía no estuvo relacionado con una insuficiente competencia en este área: I. M. Lotman conocía muy bien la filosofía en general y especialmente la filosofía europea de los siglos XVII-XIX, pero sus primeros trabajos científicos tenían no tanto un carácter filosófico cuanto un carácter interdisciplinar, situados en áreas limítrofes entre la crítica literaria, la historia y la filosofía. Sin embargo, también en estos trabajos la filosofía fue objeto, pero no método de investigación; pero cuando en los años $60 \mathrm{I}$. M. Lotman elaboró un enfoque fundamentalmente nuevo, se esforzó por evitar alusiones filosóficas. Y tan sólo un poco antes de su muerte se permitió algunas generalizaciones, próximas al discurso filosófico.

2.0. Cuando, en 1964, salieron sus Lecciones de poética estructural, que habían sentado las bases de la escuela de Tartu, y los números de Trabajos sobre los sistemas de signos que le siguieron después, en seguida quedó claro que eran percibidas hostilmente no sólo por los burócratas de la filología oficiosa, sino también por numerosos autores serios. Para que sea totalmente evidente que la cuestión residía aquí no sólo en la coyuntura política ${ }^{4}$, es suficiente citar los nombres del más eminente filósofo soviético A. F. Losev, del más grande filólogo soviético M. M. Bajtín y de autores cercanos a ellos, que mantuvieron una actitud atenta - por no decir hostil- hacia las ideas de la escuela semiótica de Tartu-Moscú ${ }^{5}$. El enfoque mostrado en las Lecciones

3 Y. M. Lotman. «Kultura kak subekt i sama-sebe objekt». In: Wiener slawistischer Almanach, Bd. 23. 1989.

${ }_{4}$ Aunque Losev y Bajtín en su juventud pagaron su tributo al marxismo, en los años 60-70 fueron percibidas si no como figuras opuestas, sí por lo menos como figuras alternativas a la ciencia oficiosa.

5 Bajtín tuvo una actitud benevolente hacia las publicaciones de Tartu, pero, más tarde, principalmente bajo la influencia del medio que le rodeaba, revisó su actitud. La actitud de la Escuela de Tartu hacia Bajtín fue, por el contrario, siempre positiva; basta con recordar que le dedicaron a él el sexto número de Trabajos sobre los sistemas de signos. En las páginas de las ediciones de Tartu tan sólo en una ocasión aparece una crí- 
resultó en principio inaceptable para la tradición humanística rusa. Para comprender las razones de esta disensión conviene detenerse, aunque sea brevemente, en su descripción.

2.1. La mentalidad rusa, en general, y la cultura humanística, en particular, percibió de forma profunda y orgánica las ideas de la filosofía romántica alemana: Schelling, Fichte, y, en primer lugar, Hegel. Durante el período soviético, el hegelianismo, como «fuente» del marxismo, se convirtió en parte de la ideología oficial, pero también antes de la revolución la mayoría de los pensadores rusos un tanto significativos (con muy pocas excepciones: N. Feodorov, V. Rozanov, P. Florenski, L. Shestov), revelan una profunda relación -a veces muy inesperada- con el sistema de Hegel. Además, las diferencias políticas y de otro tipo no desempeñan prácticamente ninguna función: el hegelianismo constituye una parte orgánica de la concepción del mundo de los eslavófilos y occidentalistas, pensadores religiosos y ateístas, revolucionarios y reaccionarios. Se puede incluso afirmar que en ningún sitio más (incluida Alemania) la pasión por Hegel fue tan grande como en Rusia. Este hecho, ya de por sí curioso, se convierte en especialmente significativo sobre el fondo de una disconformidad tan grande con otro gran filósofo alemán, Kant. «La idea rusa experimentó una extraña indeosincrasia hacia la filosofía de Kant», afirma A. V. Ajutin en un ensayo con el curioso título de Sofía y el diablo (donde Sofía es esta idea filosófica rusa, y el diablo, se supone, Kant) ${ }^{6}$. No se puede decir que las ideas de Kant pasaran inadvertidas en Rusia, pero su interpretación trasladaba constantemente esta problemática gnoseológica al campo de la ontología.

O. Mandelshtam notó de alguna forma que la poesía rusa se había intoxicado de Ariosto y Tasso, debido a que no había recibido la vacuna previa de Dante. Parafraseando a Mandelshtam, se puede decir que la desgracia de la filosofía rusa reside en el hecho de que cayó bajo los encantos de Hegel, sin haber pasado previamente por la preparación en la escuela de Kant ${ }^{7}$.

tica hacia las ideas de Bajtín: en un breve comentario de M. L. Gasparov, dirigido no tanto contra el mismo científico como cuanto contra sus discípulos.

6 A. V. Ajutin sabe de lo que escribe: pese a los intentos de parecer objetivo, este ensayo está totalmente lleno de la misma indiosincrasia. Antes de A. V. Ajutin la acusación más mordaz a Kant fue hecha antes incluso de la I Guerra Mundial por el filósofo-neoeslavófilo V. Erna, que tituló su panfleto antigermano Ot Kanta $k$ Kruppu (De Kant a Krupp). Este no es el lugar para la polémica con A. V. Ajutin, sin embargo, es muy buena aquella Sofía para la que Kant es el demonio.

1 Lo dicho, por supuesto, puede ser representado con una conocida simplifcación: el fundador de la filosofía rusa V.S. Soloviov pasó por la escuela kantiana, muchas ideas 
I. M. Lotman fue kantiano ${ }^{8}$. Aunque las notas sobre las ideas y obras de Kant se encuentran en él con poca frecuencia (por cierto, las más importantes de ellas se contienen en los trabajos de los últimos años), a lo largo de muchos años Kant fue su constante interlocutor, $y$, en las conferencias de I. M. Lotman, el nombre del pensador koenigsbergiano se recordaba con bastante más frecuencia que en los textos escritos. Pero incluso no se trata sólo de esto. Las principales teorías de la escuela de Tartu revelan una base kantiana clara.

3.0. Me detendré solamente en un ejemplo de influencia profunda de las ideas de Kant en la formación del paradigma del estructuralismo de Tartu. Su categoría central -el texto- no puede ser comprendido adecuadamente fuera de la epistemología kantiana. El texto es la cosa en sí (Ding an sich). Conviene hacer notar que la cosa en sí kantiana fue importante para el proceso de formación de la metodología de las investigaciones cibernéticas en 1950 y principios de 1960. Para la investigación de un sistema complejo fue elaborado el método de la «caja negra»: sabemos lo que llega a la entrada del sistema y lo que se obtiene a su salida, pero por principio no podemos mirar en su interior ${ }^{9}$. Estructuras análogas se hicieron populares también en las investigaciones semióticoestructurales. Compárense los modelos «SENTIDO $\Leftrightarrow$ TEXTO» (I. Melchuk) en la lingüística y «TEMA $\Leftrightarrow$ TEXTO» (A. Zholkovski e I. Scheglov) en la poética. Por ejemplo, en el modelo «SENTIDO $\Leftrightarrow$ TEXTO» se supone que el lenguaje natural es esa caja negra a cuya entrada se presentan los sentidos, y a la salida se reciben los textos equivalentes a estos sentidos.

Todas estos postulados en la lingüística estructural y en la poética conviene interpretarlos sobre el fondo del paradigma saussureano (neo)platónico: la lengua (langue) es algo primario y absoluto (la idea platónica), mientras que el habla (parole) es solamente un reflejo imperfecto, la sombra de la lengua. Pero el texto es una variedad del habla. La paráfrasis cibernética del problema, según la cual la correla-

de Kant encontraron en la cultura rusa una resonancia viva (véase también más abajo sobre P. Florenski). Pero precisamente el carácter de reflexión de las ideas de Kant muestra también la inadecuación de sus percepciones: una cosa son las discusiones sobre el imperativo categórico, etc., otro tema totalmente distinto es el análisis crítico de las bases del conocimiento filosófico. La problemática epistemologica de la filosofía de Kant se interpreta en Rusia en clave ontológica.

8 Una extraña impresión causa el intento reciente de presentar el platonismo como base de la semiótica de Lotman: R. Vetik. «Planonism of J. Lotman». In: Semiotika V. 99, \#1/2 (1994).

${ }_{9}$ Compárese, por ejemplo, U. R. Eshbi. Vvdenie v kibernetiku. Moscú, 1959. 
ción lengua/habla es la correlación entre el código (code) y el mensaje (message) solamente fortalece lo secundario del texto en relación con la lengua ${ }^{10}$. El estructuralismo se distingue de las tendencias de la lingüística que le precedieron no tanto por sus ideas y métodos nuevos, cuanto por la sustitución del objeto de investigación: para el estructuralista, la lengua no es totalmente lo mismo que, digamos, para el neogramático. El estructuralismo parece como si limpiara su parcela de investigación, quitando todo lo sobrante.

3.1. Una de las sentencias preferidas de I. M. Lotman proclama: «la piedra que rechazaron los albañiles, se hizo piedra angular» (Salmos $118,22)^{11}$. El texto era «la piedra rechazada» del estructuralismo; I. M. Lotman lo hace piedra angular de la escuela de Tartu. A diferencia del estructuralismo clásico, para la escuela de Tartu el texto no es una realidad dada directamente, sino, al igual que la lengua, es un problema, la caja negra, la cosa en sí. El texto es absolutamente inmanente en relación con la actividad extratextual, se encuentran en relaciones de complementariedad. El texto es una estructura cerrada y autónoma, y precisamente como tal debe investigarse. Elementos aislados de esta estructura no tienen un valor autónomo; su valor (el valeur saussureano) se determina por un conjunto de funciones estructurales. De aquí el empeño por elaborar una metodología de análisis inmanente del texto (método de oposiciones semánticas) en los trabajos de $1960^{12}$. Pero de aquí surge esa determinada desconfianza hacia las investigaciones en el campo de la intertextualidad: por cuanto cada elemento de la estructura del texto se caracteriza por su lugar en esta estructura, la semejanza material (o incluso la identidad) de los fragmentos de diferentes textos resulta poco significativa ${ }^{13}$.

10 Es preciso recordar también el intento de L. Hjelmslev de construir una metodología del estructuralismo sobre una base no platónica sino aristotélica (es curioso que, tras la glosemática en general y tras Hjelmslev en particular, se fortaleciera la fama del más difícil, sino el más incomprensible estructuralista, cuando en realidad se trata de la construcción del sistema más cercano al sentido común).

11 A I. M. Lotman le gustaba citar esta sentencia del apócrifo Evangelio de Tomás, donde la formulación es aún más expresiva: “iMuéstrame la piedra que los albañiles han desechado! Es la piedra angular».

12 Conviene mencionar la diferencia fundamental entre el método de construcción de series de oposiciones en los trabajos de Lotman y el método de la Escuela de Praga y, en menor medida, el de C. Levi-Strauss: las oposiciones fonológicas y morfológicas en la Escuela de Praga y las oposiciones semánticas en Levi-Strauss sirven para la descripción de la lengua, pero no para la del texto.

13 En las investigaciones que siguieron a la publicación de Lecciones de poética estructural, I. M. Lotman prestó más atención a lo que en las Lecciones llamó «relaciones extratextuales» (véase más abajo). Sin embargo, estas relaciones se estudiaron no en 
3.2. Sin embargo, la idea de inmanencia absoluta de la estructura del texto no sólo no anuló la cuestión sobre la relación del texto con la realidad extratextual, sino que, al contrario, la planteó todavía de forma más aguda. Ya Las lecciones de poética estructural concluye con una amplia sección dedicada a las relaciones extratextuales, que no generalizan lo dicho en las páginas anteriores, sino que esbozan las perspectivas de posteriores investigaciones.

3.2.1. Lo primero que fue objeto de revisión fue la correlación entre lengua y habla, fundamental para el estructuralismo. Desde el punto de vista del estructuralismo de Tartu, no se puede hablar de la supremacía de la primera sobre la segunda; además, no se puede hablar de la lengua y el habla por separado. En realidad, nos referimos a algo esencialmente distinto, por ejemplo, a la oposición glosemática (a fin de cuentas, aristotélica) de forma y sustancia, ligadas entre ellas por una relación de interdependencia. Para I. M. Lotman aquí lo más importante, en primer lugar, es el aspecto dinámico del problema ${ }^{14}$ : el engendramiento del texto no es una realización automática de los potenciales de la lengua, sino una traducción, y la tensión dinámica entre la lengua y el texto resulta una de las fuentes de evolución del sistema semiótico.

3.2.2. Después fue objeto de revisión el esquema jakobsoniano de la comunicación ${ }^{15}$, en el que la lengua y el texto funcionaban como dos de los seis componentes:

$$
\begin{gathered}
\text { código (lengua) } \\
\text { comunicación (texto) }
\end{gathered}
$$

remitente destinatario

contexto

contacto

términos de la correspondencia entre diferentes textos, o, incluso sus fragmentos, sino como relaciones estructurales en el espacio general de la cultura, estudiado como un todo. La posterior generalización de este enfoque conduce a la formación de la concepción de la semiosfera.

14 La dinámica del sistema sígnico es uno de los conceptos claves de la Escuela de Tartu, que en gran medida corrige la oposición saussureana de sincronía y diacronía (aquí, como en muchos otros aspectos, se observa una evidente relación de la escuela de Tartu con el círculo lingüístico de Praga y con los trabajos más tardíos de $\mathrm{R}$. $\mathrm{O}$. Jakobson). La dinámica en el concepto de I. M. Lotman en parte se anticipa a la idea del entonces naciente generativismo, pero en parte fue polémicamente dirigida contra la idea de N. Chomsky, cuyo generativismo, en opinión de I. M. Lotman, no estaba relacionado con la dinámica original.

15 R. Jakobson. «Closing statement on linguistics and poetics». In: T. Sebeok (ed.). Style in language. Cambridge (Mass.), 1960. 
Según R. Jakobson el remitente, teniendo en cuenta el contexto, formula con ayuda de la lengua un mensaje, que con la presencia del contacto trasmite al destinatario. Pero, según I. M. Lotman, el acto de la comunicación no es en absoluto una transmisión de un mensaje ya elaborado: no sólo la lengua no es posible antes y fuera del texto, lo mismo ocurre también con los demás componentes jakobsonianos. El contexto es el cotexto (con-texto), no puede existir antes del texto, ni en la misma forma en la que el texto depende del contexto y el contexto depende del texto. El acto de la comunicación es el acto de la traducción, el acto de la transformación: el texto trasforma la lengua, al destinatario ${ }^{16}$, establece el contacto entre el remitente y el destinatario, transforma al propio destinatario. Además, el texto se trasforma él mismo y deja de ser idéntico a sí mismo.

4.0. Si los postulados iniciales de la escuela de Tartu, que se formaron en los años 60, referentes a la estática de los sistemas semióticos, se construyen sobre la base kantiana, su desarrollo en los años 70 , que puso como piedra angular su dinámica, descubre ya otras bases filosóficas. La concepción según la cual el texto resulta diferente de sí mismo -incluyéndose (por ejemplo, en el proceso de comunicación) en todas las nuevas relaciones extratextuales, su estructura se complica constantemente, y la semántica se enriquece- obliga a recordar el logos autocreciente en Heráclito ${ }^{17}$. Aquí quiero referirme a una comparación con las ideas de F. Schiller y W. Humboldt, bastante menos eficaz, pero no por ello menos importante ${ }^{18}$.

I. M. Lotman consideraba a Schiller (a mi entender un poco exageradamente) un eminente pensador que había complementado y desarrollado de forma esencial el sistema de Kant, introduciendo en él una dimensión complementaria: la libertad y las categorías de dinámica y creación relacionadas con ella. Esto, en opinión de I. M. Lotman, creó las premisas para la superación de la antinomia sujeto y objeto, de esta maldición de la gnoseología europea. La cosa en sí resultó estar dotada de libertad creadora.

16 Compárese el artículo de I. M. Lotman «Tekst i struktura auditorii» (Trudy po znakovym sisteman 9; TRÜ toim. 422), Tartu, 1977.

17 Después de la conclusión de este trabajo, descubrí el índice y parte del texto del compendio de artículos de I. M. Lotman Logos autocreciente, que incluía tanto publicaciones ya conocidas como artículos destinados especialmente para ese volumen. Por cuanto el índice tenía en cuenta claramente material ya preparado (y no un plan para el futuro), y el mismo texto es la segunda copia mecanografiada, es posible que falten otras partes.

18 Por lo que yo sé, I. M. Lotman no cita a W. Humboldt como filósofo. Sin embargo, hay que recordar que Humboldt influyó en el pensamiento filosófico ruso tanto directamente como gracias a la mediación de A. A. Potebniá. 
En el campo del conocimiento plenamente humanístico, W. Humboldt expuso ideas parecidas, al proponer diferenciar la lengua como

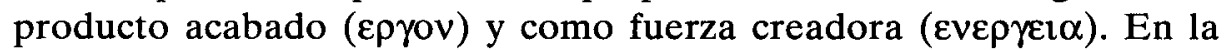
tradición estructuralista, la concepción de Humboldt se interpretaba de tal forma que al ergon le corresponde el habla (texto), y a la energeia, la lengua; la base creadora de la actividad lingüística (el langage saussureano) está concentrado en la lengua. Esta idea de Humboldt fue importante también para el generativismo, a partir de ella construye N. Chomski, en gran medida, su crítica del estructuralismo.

4.1. La originalidad del enfoque de I. M. Lotman consiste en que para él evepyeio es una propiedad, en primer lugar, del texto y no de la lengua. Esta idea, a primera vista bastante extraña, encuentra paralelo en la estética de Pavel Florenski, uno de los pensadores rusos más interesantes de principio del siglo Xx. P. Florenski subrayaba que la obra de arte (es decir, el texto del arte) es simultáneamente epyov y

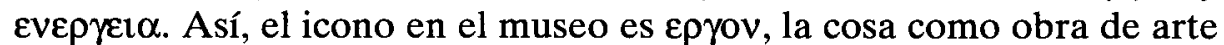
está muerta; pero en el templo ya es $\varepsilon v \varepsilon \rho \gamma \varepsilon i \alpha$. De esto P. Florenski saca la conclusión de la necesidad de crear museos de nuevo tipo: en lugar de museos de colecciones de ergones deben crearse museos que contribuyan al autodesarrollo de la energeia de las obras de arte ${ }^{19}$. Desde el punto de vista del estructuralismo de Tartu, la diferencia entre el icono en el museo y en el templo consiste en que en el museo se halla en un contexto ilimitado, rompiendo las relaciones extratextuales imprescindibles para su funcionamiento normal. Sin embargo, decir que el icono en un museo está totalmente muerto sería una simplificación: el mismo texto en gran medida crea su propio contexto; posee la capacidad de regenerar las relaciones extratextuales perdidas, de crear nuevas en lugar de las perdidas. El icono en el templo y el icono en el museo indudablemente viven una vida diferente, y las diferencias funcionales conducen a una divergencia estructural ${ }^{20}$; sin embargo, se puede hablar de la muerte de una obra artística solamente

19 Los trabajos y las ideas de Pavel Florenski estuvieron muy cercanas a la escuela de Tartu y no es casual que, precisamente, en las ediciones semióticas de Tartu fueran publicados por primera vez algunos de sus trabajos más importantes. Conviene decir que $\mathrm{P}$. Florenski queda un poco aislado entre los pensadores rusos de principios de siglo: dirigen hacia él críticas tendenciosas tanto los ateístas marxistas como los autores ortodoxos (G. Florovski, V. Zenkovski). Me parece que la causa es su kantianismo. Aunque muchas ideas de Kant fueron para P. Florenski totalmente inaccesibles, el método de su obra principal, Stolp i utverzhdenie istiny, se mantiene consecuentemente en la línea de la filosofía crítica de Kant.

20 I. M. Lotman, A. M. Piatigorski. «Tekst i funktsia». V kn.: I. M. Lotman. Izbrannye stati, T. 1, Tallinn, 1992. 
en el caso de que su destrucción física completa vaya acompañada de su destrucción completa en la memoria de la cultura. Así, el capítulo X de Evgueni Onegin quemado por Pushkin sigue viviendo en la memoria de la cultura rusa, dando señales de vida, a veces bastante curiosas.

5.0. Estos breves comentarios no tienen pretensiones de totalidad ni de sistematicidad. Su objetivo es otro: situar el paradigma conceptual de la escuela de Tartu en una determinada perspectiva filosófica. El hecho de que el descubrimiento de esa perspectiva resulte un asunto en absoluto sencillo se determina en gran medida por la actitud del fundador de la Escuela de Tartu hacia la filosofía. En conversaciones privadas ni una sola vez insistió en que la filosofía profesional sea, en nuestros días, un anacronismo (se refería a la filosofía occidental, hablar de la soviética no tenía ningún sentido). El filósofo profesional, en expresión suya, es un probador profesional de especias. El tiempo de la filosofía especulativa pasó ya, la especia debe darse con el plato principal. En relación con el saber científico, esto significa que el edificio científico no debe levantarse sobre unos determinados cimientos filosóficos formulados anteriormente, sino que la filosofía de la ciencia sólo es posible a partir de la generalización de los resultados recibidos. 